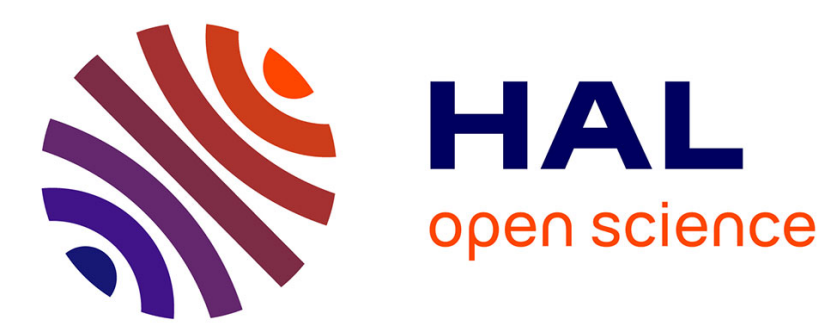

\title{
High-frequency modeling of a current transformer by finite-element simulation
}

Patrick Poulichet, F. Costa, E. Laboure

\section{To cite this version:}

Patrick Poulichet, F. Costa, E. Laboure. High-frequency modeling of a current transformer by finite-element simulation. IEEE Transactions on Magnetics, 2003, 39 (2), pp.998-1007. 10.1109/tmag.2003.808603 . hal-01870529

\section{HAL Id: hal-01870529 \\ https://hal.science/hal-01870529}

Submitted on 7 Jan 2020

HAL is a multi-disciplinary open access archive for the deposit and dissemination of scientific research documents, whether they are published or not. The documents may come from teaching and research institutions in France or abroad, or from public or private research centers.
L'archive ouverte pluridisciplinaire HAL, est destinée au dépôt et à la diffusion de documents scientifiques de niveau recherche, publiés ou non, émanant des établissements d'enseignement et de recherche français ou étrangers, des laboratoires publics ou privés. 


\title{
High-frequency modeling of a current transformer using Finite Element Simulation
}

\author{
P. POULICHET ${ }^{1}$, F. COSTA ${ }^{2}$ Member IEEE, E. LABOURÉ ${ }^{2}$
}

\begin{abstract}
This paper depicts a method to elaborate a high frequency $(100 \mathrm{kHz}$ to $50 \mathrm{MHz})$ model of a current transformer. Finite-element analysis is employed to determine the values of the different parameters of the circuit model. All electrostatic and magnetic couplings are taken into account and calculated with the finite-element method. The paper shows how parasitic capacitance and flux leakage alter the transformer frequency response. These effects are deeply modified by the primary conductor position, by an air-gap in the core and by the presence of a shielding case around the transformer. These aspects are pointed out in the study. Simulation results are compared and discussed with experimental ones in order to validate the accuracy of the models.
\end{abstract}

Index Terms--Air-gaps, Current transformers, Electromagnetic shielding, Finite element methods, High frequency modeling.

\section{INTRODUCTION}

The area of power electronics requires high performance current transformers for the following reasons:

- Static converter's switching frequency extends from $100 \mathrm{~Hz}$ to $1 \mathrm{MHz}$ so the high cut-off frequency of current transformers has to be extended to over $10 \mathrm{MHz}$.

- Current shape can be sinusoidal or pulsed containing a DC component or high frequency ringing.

- $\quad$ MOSFET or IGBT are generating high $\mathrm{dI} / \mathrm{dt}$; values up to several $\mathrm{kA} / \mu \mathrm{s}$ are usually reached. So, static converters generate strong radiated and conducted EMI [1]-[2].

This paper aims to explain how parasitic effects alter the ideal frequency response of a current transformer, and how to build a high-frequency accurate model. Therefore, improvements can be brought up in the design of a passive current transformer.

Manuscript received July 23, 2002

This work was done at the lab SATIE, SATIE is the new name of the LESIR (Lab of Electricity, Signals and Robotics).

P. POULICHET is with the ESIEE (Ecole Supérieure d'Ingénieurs en Electronique et Electrotechnique), 93162 Noisy Le Grand, France (phone: (33)1.45.92.67.18, e-mail: patrick.poulichet@esiee.fr).

F. COSTA, is with the lab SATIE, ENS (Ecole Normale Supérieure) de Cachan, 61 AV. du $\mathrm{P}^{\mathrm{t}}$ Wilson, 94230 Cachan, France (phone: (33)1.47.40.29.83 e-mail: francois.costa@ satie.ens-cachan.fr).

E. LABOURÉ is with the labSATIE, ENS (Ecole Normale Supérieure) de Cachan, 61 AV. du $\mathrm{P}^{\mathrm{t}}$ Wilson, 94230 Cachan, France (phone: (33)1.47.40.21.08 e-mail: eric.laboure@ @atie.ens-cachan.fr).
Many papers discuss how to establish the high-frequency model of a magnetic device. References [1]-[2] highlight the problems encountered when conducting current measurements in a power electronic device. They depict the realization of a high-frequency current probe $(50 \mathrm{~A}-300 \mathrm{MHz})$. However, the high-frequency electric models presented in these papers are not accurate enough to represent the high-frequency behavior of the current sensors. References [6]-[7] describe the modeling of a high-frequency voltage transformer. These papers develop equivalent circuits for a high-frequency transformer. But the models of the high-frequency phenomena are not accurate. Reference [4] describes the modeling of an induction machine stator windings using a Finite Element Method (F.E.M). The method described in this paper is interesting but the model that takes into account all the couplings between all the conductors is very complex and nonsuitable to solve our modeling problem. Reference [5] relates to the problem of the mutual inductance that depends on the primary winding position when designing a high precision mutual inductor. This paper is valuable for handling the decentred primary conductor case. In general, these mentioned papers give partial answers to our modeling objective. Therefore, a synthesis work has been necessary in order to achieve the modeling of the high-frequency behavior of a current transformer.

This work is organized as follows. Section II introduces the principle of the current transformer. Section III describes our modeling principle based on a Finite Element analysis and presents the different steps in order to construct the lumped equivalent model necessary in circuit simulations. The lumped element model will be used in order to analyze the effects of the primary conductor position in the transformer window. Finally, the model will be enhanced in order to take into account the shielding case around the transformer. Simulations of frequency response and experimental results are presented and discussed in section IV.

\section{II.PRINCIPLE OF THE CURRENT TRANSFORMER}

The current transformer principle is based on Ampere's Law as shown in Fig 1. The current transformer used in this study is realized with a toroïdal ferrite core wound with $\mathrm{N}_{2}$ secondary turns and loaded by a $10 \Omega$ resistor. An air-gap has been cut in the core in order to place a Hall sensor for DC measurement. The low cut-off frequency is expressed by: 
$f_{0}=\frac{1}{2 \pi} \frac{R}{L}$

when $\mathrm{L}$ is expressed by

$L=\frac{\mu_{0}}{t} N_{2}^{2} A_{e}$

$A_{e}$ is the area of the magnetic circuit, $t$ is the air-gap width. The main characteristics of the current transformer are presented in Table I. In this configuration, the low cut-off frequency is situated around $1 \mathrm{kHz}$.

Fig. 1. Description of the current transformer

TABLE I

MAIN CHARACTERISTICS OF THE CURRENT TRANSFORMER

The air-gap avoids the magnetic core to be saturated by a DC component in the primary current. Furthermore, it increases the linearity of the magnetic curve.

The main effects that influence the high-frequency limitations are:

- The capacitance between the primary and the secondary windings. For example, if the primary conductor is situated in the vicinity of a secondary turn (see Fig. 2), the capacitive coupling between this turn and the primary conductor is increased.

- The resonance between the transformer leakage inductance and the capacitance located between the secondary winding and the core.

- The variations of electromagnetic couplings in the transformer due to the position of the primary conductor in the transformer window.

Fig. 2 shows the magnetic core inserted into a shield. An air-gap in the top of the shield is used in order to avoid a shortcircuit. This air-gap is situated between the primary conductor and the magnetic core. Outside the core window, the shielding case is closed. It has been shown in [2] that the window part of the shield reduces the flux leakage in the high-frequency range $(100 \mathrm{kHz}-10 \mathrm{MHz})$.

Fig. 2. Primary conductor located near the air-gap and representation of the shield around the current transformer

\section{A. Low frequency model of the transformer}

A description of the low-frequency model of the transformer used in the later part is depicted. The electric model derived from the fundamental equations of a magnetic device is presented in Fig. 3.

Fig. 3. Electric circuit model for a generic two winding transformer

When $\mathrm{k}$ is defined as the coupling coefficient, the turn ratio of the transformer is evaluated with (3). $\mathrm{n} 1$ is the turn number of the primary and $\mathrm{n} 2$ is the turn number of the secondary.

$$
m=k \frac{n_{2}}{n_{1}}
$$

The model of the transformer is defined if $L \mu$, If and $m$ are determined.

\section{B. High frequency model of the transformer}

The high frequency limitation of the current transformer transfer function depends on two main factors:

- Firstly, the coupling capacitance between the primary and the secondary winding causes a resonance with the leakage inductance, and it causes the transfer function to raise up at the resonant frequency.

- Secondly the differences in the values of each section's coupling coefficient alters the transfer function. It causes successive resonance and anti-resonance with large variations of the transfer function.

These effects will be highlighted in the following part. Simple models will be used later in order to explain the experimental results.

Reference [2] shows the influence of the capacitive coupling between the primary and secondary windings. This effect on the transfer function is caused by the resonance frequency due to the leakage inductance of and to the capacitances $\mathrm{Cp}$ (primary winding capacitance), Cps (primary to secondary winding capacitance) and Cs (secondary winding capacitance). At resonance conditions, the transfer function level increases strongly. The first resonance frequency is expressed by (4).

$f_{1}=\frac{1}{2 \pi \cdot \sqrt{l_{f} \cdot m \cdot\left(C_{p}+C_{p s}\right)}}$

Now if we assume that the coupling factor, the leakage inductance and the capacitive coupling are not uniformly distributed, a simplified model as depicted in Fig. 4 can be used. Only two sections have been considered. Fig. 4 highlights the phenomena which alters the transfer function. A resonance between the leakage inductance lf and the secondary capacitance $\mathrm{C}$ appears when the two coupling coefficients $\mathrm{m} 1$ and $\mathrm{m} 2$ are different. Two resonances can be observed depending on the leakage inductance, the capacitance values $\mathrm{C}$ and the coupling coefficients $m$ values.

Fig. 4. Two resonance frequencies caused by the non-uniformly distributed coupling values

In this simple case, the resonance frequency $f_{2}$ is calculated by (5),

$f_{2}=\frac{1}{2 \pi} \sqrt{\frac{M}{\frac{C}{2}\left(l_{1} \cdot \frac{m_{2}}{m_{1}}+l_{2} \cdot \frac{m_{1}}{m_{2}}\right)}}$ with $M=\left(1+\frac{m_{2}}{m_{1}}\right)\left(1+\frac{m_{1}}{m_{2}}\right)$ (5)

and the anti-resonance frequency $\mathrm{f}_{3}$ is calculated by (6).

$f_{3}=\frac{1}{2 \pi} \sqrt{\frac{1}{l_{1} \cdot \frac{C}{2}}\left(1+\frac{m_{1}}{m_{2}}\right)}$ 


\section{MODELING PRINCIPLES}

The determination of all parameters of the high-frequency equivalent model using the F.E.M. method is described.

A modeling method for magnetic circuits has been introduced in [9] in order to predict the distribution of the leakage energy in the windings of a magnetic structure. This method is not applicable in the case of a partially wound core because of the air-gap.

The method based on F.E.M. analysis is presented in order to determine the elements of the model. Afterwards, a description for determining the inductive and capacitive parameters is described.

\section{A. Determination of the parameters of the transformer model}

Consider the model presented in Fig. 3. The parameters can be obtained with the following process.

\section{1) Determination of the magnetizing inductance $L_{\mu}$}

In a first step, a $1 \mathrm{~A}$ current is applied in the primary winding and the value of $L \mu$ is obtained when calculating the magnetic energy $\mathrm{W}_{1}$ in the volume of the core and in the air around the core:

$\mathrm{W}_{1}=1 / 2 \mathrm{~L}_{\mu}$

\section{2) Determination of the turn ratio $m$}

In a second step, a $1 \mathrm{~A}$ current is applied in the secondary winding of the model presented in Fig. 3. The energy stored in the system is:

$\mathrm{W}_{2}=1 / 2\left(\mathrm{~L}_{\mu}+\mathrm{lf}\right) \mathrm{m}^{2}$

In a third step, a $1 \mathrm{~A}$ current is applied in the primary and in the secondary. So, it is equivalent to applied $1 \mathrm{~A}$ in the primary and $1 \mathrm{~A}$ in the secondary of Fig. 3. The energy $\mathrm{W}_{3}$ in all the materials of the F.E.M. simulation is given by:

$\mathrm{W}_{3}=1 / 2\left(\mathrm{~L}_{\mu} \mathrm{m} .(\mathrm{m}-1)+\right.$ lf. $\left.\mathrm{m}^{2}\right)$

The 3D F.E.M. technique is used to calculate the energy $\mathrm{W}_{1}$, $\mathrm{W}_{2}$ and $\mathrm{W}_{3}$. Using relations (7) (8) and (9), (10) is obtained in order to determine the turn ratio $\mathrm{m}$.

$m=\frac{W_{2}-W_{3}}{W_{1}}$

\section{3) Determination of the global leakage inductance}

A $1 \mathrm{~A}$ current supplies the primary conductor. Each part of the secondary winding (see Fig. 2) is supplied by a 1A current divided by the number of turns, in order to obtain zero-flux in the magnetic circuit. The value of lf is obtained when calculating the magnetic energy $\mathrm{W}_{4}$ in all the materials.

$\mathrm{W}_{4}=1 / 2$ lf

The partial leakage inductance Ifi (associated with each section around the core) is obtained with the energy calculated in the volumes around a turn.

\section{B. Determination of the circuit model}

The modeling principle shown in Fig.5 consists of dividing the winding into sections, each one is represented by an equivalent circuit. The current transformer is divided into sections (Fig. 5.a). Each section is represented by a single turn placed on the core for F.E.M. analysis (Fig. 5.b). All sections are assembled for establishing a circuit model of the current transformer (Fig. 5.c). The elements of each section are determined analytically or measured when possible or evaluated by F.E.M. simulation as previously described. The variation of the resistance of the secondary winding with the frequency is represented by the block "skin effect" of Fig. 5.c. It is determined with F.E.M. simulation.

Fig. 5. Transformer sections and associated electrical models

The electrostatic and magnetic energy situated around each turn (i.e. a section) permits us to obtain the parameters of each section electrical circuit. As a consequence, the values of the section's components may be different relative to the primary winding position or to the air-gap vicinity. For example when the primary conductor is in the vicinity of one section, the capacitance $\mathrm{Cps}$ of the nearest section is greater than the ones of the opposite side. In order to take into account the capacitive couplings between the secondary winding and the poorly conducting ferrite core, the impedance $\mathrm{Zw}$ is introduced in each section.

Based on results determined for 5 sections of Fig. 5, new parameters have been interpolated in order to establish a model with 10 sections, corresponding to 100 turns (10 sections of 10 turns each) transformer. This method allows us to have a sufficient accuracy and to limit the simulation time. When all the components of the 10 sections are determined, the global model of the current transformer is constituted as shown in Fig. 6. In the primary side, all the connections of the sections are connected in parallel on the global magnetizing inductance $\mathrm{L} \mu$. In the secondary side, the sections are connected in series. All sections are connected together by an impedance named $Z_{c o r e}$. It models the impedance of the core. It has been measured as described in paragraph III.E.

Fig. 6. Connection of all sections in order to constitute the model of the current transformer 


\section{Determination of magnetic parameters with F.E.M. simulation}

\section{1) Magnetizing inductance $L \mu$}

Firstly, it is necessary to determine how the magnetic flied B is distributed in the core and in the air-gap. This is done by using Magnetostatics part of the software [3]. The magnetic properties of the materials and the current density in the primary conductor are included in the input data.

The sum of the energies in the air, in the conductor and in the ferrite core defines the magnetizing inductance. Because the computation volumes represent $1 / 4$ of the model, the magnetizing inductance is given by (12):

$$
L_{\mu}=\frac{8}{I_{p}^{2}} \iiint \frac{\mu H^{2}}{2} d v
$$

Table II give the calculation results for two permeability values and 1A primary current (Ip).

TABLE II. VALUE OF ENERGY IN THE DIFFERENT PARTS OF THE TRANSFORMER

The following values are obtained:

$$
\begin{aligned}
& \mu_{\mathrm{r}}=1000, \mathrm{~L}_{\mu}=203 \mathrm{nH} \\
& \mu_{\mathrm{r}}=3000, \mathrm{~L}_{\mu}=215 \mathrm{nH} .
\end{aligned}
$$

These values are close together because the energy is mainly stored in the air-gap and the $L \mu$ value remains almost constant.

The influence of the primary conductor position on magnetizing value inductance has been studied. Fig. 2 presents the axes system, which locates the position of the conductor. Table III compares the variations on the inductance $L \mu$ values measured and computed by F.E.M. at different conductor positions.

Table III. Variations of the magnetizing inductance, measured and calculated

Table III shows some differences between the simulated results and the measurements. They can be explained by the experimental configuration that differs from the ideal simulation topology. Nevertheless the results are very close. This confirms the validity of this first modeling step.

\section{2) Determination of the global leakage inductance}

In order to compute the leakage value, the eddy-current analysis module [3] is used. The leakage inductance is obtained by applying an $\mathrm{AC}($ at $1 \mathrm{MHz})$ current density in the primary and secondary conductors. The total magnetic energy in the air around the core and in the conductors corresponds to the total leakage inductance. The values of the total leakage inductance calculated for different positions of the primary conductor are indicated in Table IV. The value of the total leakage inductance is globally reflected to the primary conductor and it represents all the magnetic leakage effects of the transformer.

TABLE IV. VALUES OF TOTAL LEAKAGE INDUCTANCE REFLECTED TO THE PRIMARY

\section{3) Calculation of the partial inductance}

Partial leakage inductance is determined by the magnetic energy located around a section and in the space between the primary conductor and the section. The values are given in Table VII.

\section{4) Calculation of the coupling coefficients $m$}

In order to determine the coupling coefficient $\mathrm{m}$ for each section a current density corresponding to $1 \mathrm{~A}$ is applied successively in each section. The energy W3 of Table V is obtained when the primary conductor and a secondary section are supplied. The coupling coefficient $\mathrm{m}$ is obtained using equation (10).

\section{TABLE V. ENERGY W2 AND W3 FOR EACH SECTION}

The value of the magnetizing inductance calculated by F.E.M. is $L \mu=154 \mathrm{nH}$. This value is different from the values obtained in Table III because in that case the F.E.M. meshing has been simplified. The coupling coefficients are calculated with the values of the Table $\mathrm{V}$, and Table VI gives the $\mathrm{m}$ values of the 5 sections. The coupling coefficient $\mathrm{m}$ of section 1 (situated near the air-gap) is much smaller because the core cannot perfectly conduct the flux. Inversely, section 3 is situated in the middle of the core where the flux is better conducted. So the $\mathrm{m}$ value is close to 1 .

TABLE VI. VALUES OF M FOR EACH SECTION

\section{5) Values of $m$ and lf for the complete current transformer}

Using the values that have been previously determined, ten new values of $\mathrm{m}$ are interpolated. Each of these values corresponds to a section of ten turns, and finally the electric model of the current transformer is defined corresponding to 100 turns. The coupling coefficient $\mathrm{m}$ and the leakage inductance lf are presented in Table VII when the primary is well centered ( $x=0$ and $y=0)$, when the primary is placed near the air-gap $(x=0,01$ and $y=0)$ and when the current transformer is shielded. When the primary conductor is placed near the airgap, the coupling coefficient $\mathrm{m} 1$ increases because the primary is located near section 1. The leakage inductance If increases when the primary conductor is located near the air-gap. 
TABLE VII. TURNS RATIO $m$ AND LEAKAGE INDUCTANCE If WHEN THE PRIMARY CONDUCTOR IS WELL CENTERED $(\mathrm{X}=0, \mathrm{Y}=0)$, WHEN THE PRIMARY CONDUCTOR IS PLACED NEAR THE AIR-GAP $(\mathrm{X}=0,01, \mathrm{Y}=0)$ AND WHEN THE CURRENT TRANSFORMER IS SHIELDED.

When the current transformer is shielded (modeling of the Fig. 2) the F.E.M. calculations have shown that the values of the leakage inductance are strongly reduced and become constant. Furthermore the coupling coefficients of each section are almost constant. These results are very interesting because they indicate that the cut-off frequency increases. These characteristics are verified in the experimental results section.

\section{Electrostatic effects : determination of parasitic capacitances}

Reference [8] depicts an analytical approach based on simplified assumptions. The F.E.M. is used to determine the capacitance in a complex topology of conductors because an analytical method is not possible in this case.

As indicated in Fig. 5.c, three main electrostatic effects are included in the model of a section:

- The capacitance between the primary conductor and secondary $\mathrm{C}_{\mathrm{ps}}$, and $\mathrm{C}_{\mathrm{ps}}$ '. When the primary conductor is located near the air-gap, these capacitances are different. These values are calculated with the F.E.M..

- The capacitance between the primary conductor and the magnetic material $\mathrm{C}_{\mathrm{pc}}$, and $\mathrm{C}_{\mathrm{pc}}$ ". The influence of this capacitance is important when the primary conductor is located near the core. The values are calculated with the F.E.M.

- The capacitance between the secondary winding and the magnetic material Cs. This capacitance is uniformly distributed. This value is calculated with F.E.M. and verified experimentally.

An improved model that takes into account the shield is proposed in Fig. 7. The capacitance between the primary conductor and the secondary winding is represented by two capacitances connected together to the shield.

Fig. 7. Model of a shielded section

\section{1) Principle of capacitance calculation}

The electrostatic field simulator computes the static electric fields arising from potential differences and charge distributions. The field simulator solves the electric potential $\mathrm{V}$ in the field equation derived from the Gauss law. The electrostatic energy $\mathrm{W}$ is calculated. Because the computation volume represents $1 / 2$ of the model, the capacitance $C$ is expressed by :

$C=4 . W$

The capacitance between the primary conductor and a section $\left(\mathrm{C}_{\mathrm{ps}}\right.$, or $\mathrm{C}_{\mathrm{ps}}$, for each section) is defined in each region of space, especially when the primary conductor is not centered. This capacitance is obtained when a potential of $1 \mathrm{~V}$ is applied between the primary conductor, and successively to each section.

\section{2) Capacitance Cs and impedance $Z w$}

The capacitance between all the sections of the secondary winding and the core is determined when a potential of $1 \mathrm{~V}$ is applied between the winding and the core. This value is about $16 \mathrm{pF}$ when the thickness of the insulator is $0,1 \mathrm{~mm}$. Because this value is crucial, we have measured with a network analyzer the impedance between one end of the winding and a contact stuck on the core. Fig. 8 compares the measurement of the impedance for a 10 turns winding that represents a section and the simulation results.

Fig. 8. Equivalent impedance measurement between one output of the winding and the magnetic core

Fig. 8 shows a good agreement between the impedance measured in the case of 10 turns winding, and the impedance of the circuit model. The dipole represents the capacitive coupling Cs and the losses in the dielectric. The values of the model are given for one section composed by ten turns.

\section{3) Values of capacitance used for the simulations}

Table VIII presents the different values of the capacitances of the section models described in Fig. 5.c and in Fig. 7. Despite the Cps (primary to secondary capacitance) value being very small, it is very important to take it into account. In the case when the primary conductor is well centered, Cps and $\mathrm{Cpc}$ are uniformly distributed in each section. So the energy is computed in all the F.E.M. model and distribute with the same value. When the primary conductor is located near the air-gap, the capacitances are determined with the energy computed in the regions around a section. The curve of the value of capacitance as a function of the turn angle is constructed in order to interpolate new values of $\mathrm{Cps}$ and $\mathrm{Cpc}$ [11].

TABLE VIII. TOTAL CAPACITANCE USED FOR THE SIMULATION

\section{E. Influence of the magnetic core: electrical model of the core}

Core connections are provided in the model of the Fig. 6 in order to connect them together with the impedance Zcore that takes into account the impedance of the magnetic material. The impedance of the magnetic material core has been measured when two contacts stuck on the core are separated with the same space as a 10 turns winding. This impedance measurement is shown in Fig. 9.

Fig. 9. Impedance measurement of magnetic material

A functionally dependent model of this impedance (modulus and phase) has been included in the electrical model. However, the variation of the impedance with the frequency is 
due to the constitution of the ferrite material [10].

\section{EXPERIMENTAL RESULTS AND DISCUSSION}

The global model presented in Fig. 6 is determined for the unshielded and for the shielded current transformer. The partial model of Fig. 5.c (for the unshielded current transformer) and the partial model of Fig. 7 (for the shielded current transformer) are determined with the magnetic results of the Table VII and with the capacitance given in the Table VIII. The magnetic impedance is represented with a Spice function that represents $Z_{c o r e}$. The equivalent circuit of the Fig. 8 replace the impedance $\mathrm{Zw}$.

In this section comparisons between the simulation of the electric model depicted up to now and the measurements of the current transformer are conducted.

\section{A. Unshielded current transformer}

\section{1) Centered primary conductor}

In order to obtain accurate measurements, a specific adaptation device on the network analyzer has been built. The cable from the network analyzer that passes through the window of the current transformer is fixed far from the metal case shielding of the adaptation device. Calibration of the circuit is allowed. Fig. 10 compares the measured and simulated transfer function gain and phase versus frequency when the primary conductor is centered.

Fig. 10. Current transformer transfer function gain and phase versus frequency when the primary conductor is centered. Thick lines: Measurements. Thin line: Simulation.

A satisfactory matching can be observed between the simulation and the experimental results. The measured sensitivity of the current transformer is $83 \mathrm{mV}^{-\mathrm{A}^{-1}}$ and the sensitivity obtained with simulation is $85 \mathrm{mV} \cdot \mathrm{A}^{-1}$. The error is $2,4 \%$. The sensitivity is fixed with the sum of the partial coupling coefficient of the Table VII ( $\sum_{\mathrm{i}=1}^{10} \mathrm{~m}_{\mathrm{i}}=90$ turns $)$ and with the loaded resistance (10 $\Omega / / 50 \Omega$ ); So the sensitivity is $92,5 \mathrm{mV} \cdot \mathrm{A}^{-1}$. But the leakage inductance, the resistance of the winding and the impedance of the magnetic material contribute to reduce the sensitivity.

The usable bandwidth of the transformer is correctly calculated. The shape of the curve around the cut-off is well reproduced. Note that the capacitive coupling between the primary and the secondary windings can be observed with the behavior of the curve above the $30 \mathrm{MHz}$ frequency. As shown in Fig. 4 successive resonances and anti-resonances can be noted.

The phenomena in the range $6-8 \mathrm{MHz}$ are corresponding to the highest values of the leakage inductance and capacitance. The first anti-resonance and resonance frequencies measured are respectively:

$$
\mathrm{f}_{2}=7 \mathrm{MHz} \text { and } \mathrm{f}_{3}=7,5 \mathrm{MHz}
$$

These frequencies can be estimated with the relations (5) and (6) and with the results of the Table IX. The minimal and maximal values of the leakage inductance of the Table VII are reported to the secondary.

\section{TABLE IX. MAIN RESULTS FOR PRIMARY CONDUCTOR WELL CENTERED}

(5) and (6) gave: $f_{2}=6,47 \mathrm{MHz}$ and $\mathrm{f}_{3}=7,12 \mathrm{MHz}$. These values are close to the previous values. Obviously, the propagation phenomenon is not taken into account in our models. Therefore, the validity of the frequency analysis is limited to around 50MHz. The resonance over $40 \mathrm{MHz}$ cannot be predicted because of the restricted number of sections in the model. Another limitation is introduced by the measurement process. Because the Cps value is very small $(0,6 \mathrm{pF})$, the influence of the metal case shielding of the adaptation device cause a dependence of the current transformer transfer function gain with the placement of the air-gap.

Nevertheless two important points are correctly determined: the sensitivity of the current transformer and its frequency limitation.

\section{2) Influence of the impedance of the magnetic material}

We compares two simulations with different values for the impedance $Z_{\text {core. }}$ When the impedance $Z_{\text {core }}$ is high, the high cut-off frequency is improved. This is interesting in order to increase the high cut-off frequency of the current transformer.

\section{3) Non centered primary conductor}

Fig. 11 shows the current transformer transfer function when the primary conductor is located $1,5 \mathrm{~mm}$ near the air-gap. Significant changes can be observed in the experimental or simulated curves compared to Fig. 10.

Compared to the results with the centered primary conductor, the global leakage inductance increase from $14,7 \mathrm{nH}$ to $20 \mathrm{nH}$. Table VII shows that the coupling coefficient increase when the primary conductor is located near the secondary winding $\left(\sum_{\mathrm{i}=1}^{10} \mathrm{~m}_{\mathrm{i}}=94,6\right.$ turns $)$. The measured sensitivity is $73,6 \mathrm{mV}$.A-1 and the simulated sensitivity is $76,8 \mathrm{mV}$.A-1. The error is $4 \%$.

Fig. 11. Gain and phase of the current transformer transfer function when the primary conductor is off centered. Thick lines: Measurements. Thin line: Simulation.

The measurement shows an anti-resonant frequency at $6 \mathrm{MHz}$. The simulation show a small resonance at this frequency. Comparisons between measurement and simulation shows the permutation of the resonance and anti-resonance frequency. Consider the circuit represented in Fig. 4, the permutation of resonance frequency and anti-resonance 
frequency is achieved when a permutation between $\mathrm{m} 1$ and $\mathrm{m} 2$ is operated. The same permutation in the real coupling coefficient can explain these differences between simulation and experimental results. Table VII shows that the coupling coefficient of the section near the air-gap is increased compared to the centered primary conductor.

Note that the successive resonances over the first one depend strongly on the small capacitive couplings between the primary and secondary windings, and between the primary conductor and the core. These capacitances are difficult to evaluate and the influence of the shield around the current transformer is very important in order to limit the capacitive couplings.

\section{B. Shielded current transformer}

Table VII shows that the shielding around the core reduces the leakage inductance and makes uniform the different coupling coefficients. The global primary leakage inductance is reduced to $3,2 \mathrm{nH}$ and it is uniform. Like the shielding box is connected to ground, the capacitive coupling between the primary conductor and the secondary winding is reduced. As shown in Fig. 12, the capacitive coupling is cancelled in simulation and strongly reduced on measurement.

The high frequency limitation of the current transformer function is situated around $14 \mathrm{MHz}$. It is correctly evaluated with the simulation. The simulation shows a small antiresonance at this frequency. The frequency limit is increased from $4 \mathrm{MHz}$ when the current transformer is centered to $14 \mathrm{MHz}$ in this configuration. The measurement shows that the sensitivity is $83 \mathrm{mV}^{-\mathrm{A}^{-1}}$. The sensitivity obtained by simulation is $12 \%$ overvalued. This difference is probably due to the lack of accuracy in the modeling of the coupling coefficient.

This configuration is very interesting in order to built a high-performance (frequency-cut-off, EMI immunity) current transducer.

Fig. 12. Gain and phase of the shielded current transformer transfer function when the primary conductor is centered. Thick lines: Measurements. Thin line: Simulation.

\section{CONCLUSION}

The study presented herein depicts a mixed method based on F.E.M. and on circuit representation in order to elaborate an accurate high-frequency model of a current transformer. The calculation of the transformer elements based on F.E.M. analysis is shown. In F.E.M. simulation, the energy is calculated in a region of space and the equivalent inductance or capacitance (that depends on magnetic or electrostatic simulation) is related to the circuit model. The magnetic properties of the core are taken into account.

The origin of the reduction of the high cut-off frequency in the case of the non-centered primary conductor can be explained by non-uniformly distributed leakage inductance and the non-uniform repartition of the coupling coefficient. The interest of using a shield around the core is shown. It makes uniform the distribution of the coupling coefficient of the different parts of the winding, it reduces the leakage inductance and it reduces the capacitive coupling. As a consequence, the shielded current transformer has a higher cutoff frequency.

Thus, we have demonstrated that it was possible to optimize the design of a current sensor and to predict its high cut-off frequency.

The modeling principle is very interesting in order to optimize the design of complex winding used in flux-gate or integrated transformer. In the future, the design of integrated transformer will be facilitated with F.E.M. software that has libraries with different shape of magnetic circuit and different topologies of winding.

\section{REFERENCES}

[1] F. Costa, E. Labouré, F. Forest, and C. Gautier, "Wide bandwidth, large AC current probe for power electronics and EMI measurements", IEEE Trans. On Industrial Electronics, vol. 44, №4, pp 502-511, August 1997.

[2] E. Labouré, F. Costa and F. Forest, "Current measurement in static converters and realisation of a high frequency passive current probe (50A-300MHz)," in Proc. EPE'93, Brighton, U.K., $\mathrm{N}^{\circ} 377$, vol. 4, $\mathrm{N}^{\circ}$ 377, pp 478-783, 1993.

[3] ANSYS Electromagnetic Field Analysis Guide. 3D Magnetostatics and fundamental of edge-Based Analysis. Chapter 6. Ansys version 5.5 Research. 2000. Cril technology France.

[4] G. Suresh, H. A. Toliyat, D. A. Rendusara and P. N. Enjeti, "Predicting the transient effects of PWM voltage on the stator windings of random wound induction motor", IEEE Trans. On Power Electronics, vol. 14, pp 23-30, January 1999.

[5] Petar N. Miljanié, "Mutual inductance highly independent of primary winding position and ambient fields", IEEE Trans. on Instrumentation and measurements, vol. 46, №2, pp 471-473, April 1997.

[6] A. Ahmad, Ph. Auriol and C. Kieny, "Shell-form power transformer modeling at high frequency", IEEE Trans. On Magnetics, vol. 30, ํ5, pp 3729-3732, September 1994.

[7] J. Faiz, B. Abed-Ashtiani and M. R. Byat, "Lumped complete equivalent circuit of a coreless high-frequency transformer", IEEE Trans. On Magnetics, vol. 33, №1, pp 703-707, January 1997.

[8] G. Grandi, M. K. Kazimierczuk, A. Massarini and U. Reggiani, "Stray capacitance of single-layer solenoid air-core inductor", IEEE Trans. On Industry Applications, vol. 35, N5, pp 1162-1168, September/October 1999.

[9] Abraham Dauhajre and R.D. Middlebrook, "Modeling and estimation of leakage phenomena in magnetic circuits", in Proc. IEEE-IAS Conf., 1986, pp. 213-216.

[10] E.C. Snelling, "Soft Ferrites Properties and Applications", Second Edition Butterworth, 1988.

[11] P. Poulichet, "Modélisation, conception et réalisation de capteurs de courant performants", Doctoral thesis October 2001, Ecole Normale Supérieure de Cachan, France. 